\title{
Efficacy and Safety of Indwelling Pleural Catheters in Management of Hepatic Hydrothorax: A Systematic Review of Literature
}

Muhammad A. Baig ${ }^{1}$, Muhammad B. Majeed ${ }^{2}$, Bashar M. Attar ${ }^{3}$, Zubair Khan ${ }^{4}$, Melchor Demetria ${ }^{3}$, Seema R. Gandhi ${ }^{3}$

1. Medicine, John H Stroger J. Hospital of Cook County, Chicago, USA 2. Medicine, John H. Stroger Jr. Hospital of Cook County, Chicago, USA 3. Gastroenterology and Hepatology, John H. Stroger Jr. Hospital of Cook County, Chicago, USA

4. Internal Medicine, University of Toledo Medical Center, Toledo, USA

Corresponding author: Muhammad A. Baig, mbaig2@cookcountyhhs.org

\begin{abstract}
Hepatic hydrothorax $(\mathrm{HH})$ is an infrequent but debilitating and therapeutically challenging complication of advanced liver cirrhosis. As evidence suggests against chest tube placement in $\mathrm{HH}$, many clinicians are reluctant to place indwelling pleural catheters (IPCs) for non-malignant effusions like HH. We aim to study the efficacy and safety of IPCs as an alternative treatment option in our systematic review.
\end{abstract}

A literature search was conducted using the electronic database engines MEDLINE, PubMed, EMBASE, Ovid, Scopus and Cochrane Library (Cochrane Central Register of Controlled trials and Cochrane Database of Systematic Reviews) from inception to April 2018 to identify published articles and reports addressing outcomes in patients treated for HH with IPCs. The risk of bias was rated for each study using the Cochrane criteria.

The search strategy retrieved 370 papers, of which four case series were selected with a total of 111 patients. After the insertion of IPCs for HH, spontaneous pleurodesis was achieved in 16 (31.4\%) out of 51 patients at a mean duration of 73-222 days. As far as secondary outcomes were concerned, the frequency of pneumothorax during or after the procedure was $0(0 \%)$ out of 92 patients, pain at insertion site $12(20 \%)$ out of 60 patients, catheter blockage two (2.9\%) out of 68 patients, pleural fluid infection five (4.5\%) out of 111 patients and catheter-site cellulitis one (3.1\%) out of 32 patients. Re-accumulation of pleural fluid after catheter removal was mentioned in one study, wherein 12 (20\%) out of 60 patients developed recurrence of pleural effusion.

We conclude IPCs as an acceptable therapeutic option for the management of refractory pleural effusion in patients with HH. Although trans-jugular intrahepatic portosystemic shunt (TIPS) and liver transplantation are the gold standards for the management of pleural effusion in these patients, cost and availability are the major concerns with these treatment modalities. IPCs are a safe and efficacious alternative with a

Received 07/25/2018

Review began 08/03/2018 Review ended 08/03/2018 Published 08/06/2018

\section{(c) Copyright 2018}

Baig et al. This is an open access article distributed under the terms of the Creative Commons Attribution License CC-BY 3.0., which permits unrestricted use, distribution, and reproduction in any medium, provided the original author and source are credited.

Categories: Cardiac/Thoracic/Vascular Surgery, Internal Medicine, Gastroenterology

Keywords: hepatic hydrothorax, indwelling pleural catheters, pleural catheters, systematic review

\section{Introduction And Background}

Hepatic hydrothorax $(\mathrm{HH})$ is defined as a transudative pleural effusion $>500 \mathrm{~mL}$ in patients with cirrhosis in the absence of cardiac and pulmonary diseases [1]. In patients with cirrhosis, the incidence of $\mathrm{HH}$ is $5 \%$ to $10 \%$, and $\mathrm{HH}$ occurs typically as an isolated right-sided pleural effusion in $70 \%$ to $80 \%$ of cases. The relief of dyspnea in patients with $\mathrm{HH}$ that is refractory to conservative management remains a clinical challenge [2]. As the majority of these patients have concomitant ascites, the therapeutic aim is typically to treat the ascites with salt restriction and diuretics and provide thoracentesis as needed [3].

It can become increasingly challenging when patients develop diuretic-resistant $\mathrm{HH}$, as the only options available are liver transplantation, trans-jugular intrahepatic portosystemic shunt (TIPS) and indwelling pleural catheter (IPC). Several case series have demonstrated the association of chest tube placement in patients with HH with complications such as major electrolyte imbalances, malnutrition, severe protein loss, acute renal injury, pneumothorax and empyema [4-7]. Given the questionable safety of chest tube placement, many clinicians are reluctant to place IPCs for managing non-malignant effusions like HH [8]. Moreover, limited objective data are available on the efficacy of IPCs in this patient population.

To the best of our knowledge, this is the only systematic review of literature to date detailing the safety and efficacy of IPCs in patients with $\mathrm{HH}$. 


\section{Review}

\section{Materials and methods}

A literature search was conducted using the electronic database engines MEDLINE, PubMed, EMBASE, Ovid, Scopus and Cochrane Library (Cochrane Central Register of Controlled Trials and Cochrane Database of Systematic Reviews) from inception to April 2018 to identify published articles and reports addressing outcomes in patients treated for HH with IPCs. The combinations of keywords used were "indwelling pleural catheter" or "PleurX catheter" or "tunneled pleural catheter" or "pleural catheter" or "catheter" and "hepatic hydrothorax". The reference lists of all eligible studies were reviewed to identify additional studies.

Published studies, case series and case reports were eligible for inclusion if they reported the use of IPCs for the management of HH. Articles were excluded if (1) they were not written in English, (2) no outcomes were reported, or (3) they represented single case reports, review articles or studies published as abstracts only. In observational studies using multiple modalities for the management of $\mathrm{HH}$, data from the cohort of patients who underwent IPC placement were collected and analyzed. Two reviewers (MB and MM) independently performed study selection according to the eligibility criteria. Disagreements were resolved by discussion with a third reviewer (ZK). A Preferred Reporting Items for Systematic Reviews and Meta-Analyses flow diagram detailing the review process is shown in Figure 1.

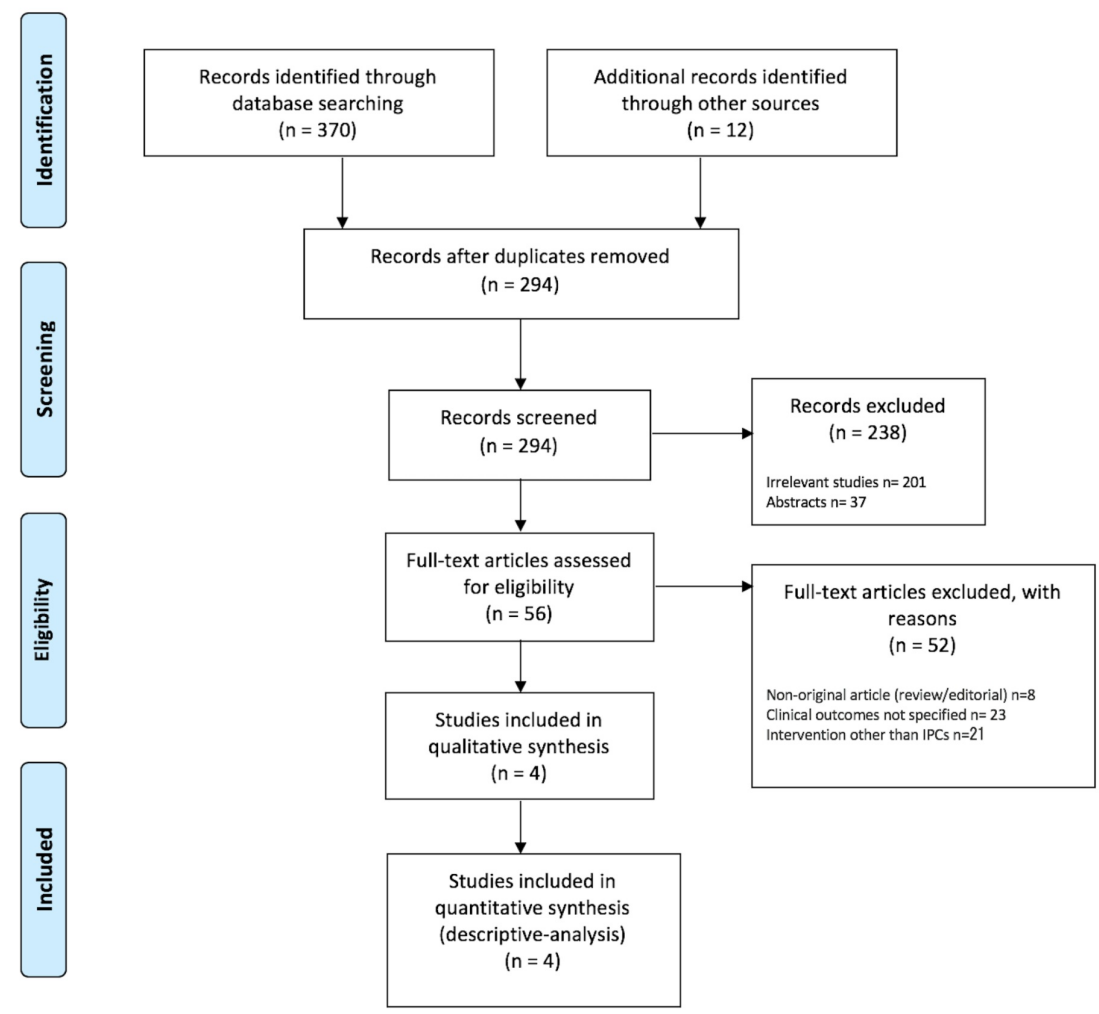

FIGURE 1: Preferred Reporting Items for Systematic Reviews and MetaAnalyses flow diagram detailing the review process.

The following data were independently abstracted into a standardized form: study characteristics (study design, primary author, time period of study, year of publication and country of the population studied), characteristics of the study population (total number of patients undergoing IPC placement, age of patients, gender, location of pleural effusion, Child-Pugh Class, Model for End-Stage Liver Disease score and etiology of cirrhosis), type and frequency of complications (pneumothorax, insertion-site pain, blockage of catheter, pleural fluid infection, re-accumulation after catheter removal, catheter-site cellulitis and dislodgement of the catheter) and outcomes of IPCs (spontaneous pleurodesis and mean duration to achieve spontaneous pleurodesis).

We systematically assessed the quality of the studies using the published criteria [9-10]. To comprehensively assess the quality of clinical studies, we used the Newcastle-Ottawa Scale (NOS) [11]. Again, in the case of a discrepancy in the quality assessment between the two study investigators (MB and MM), the senior investigator (ZK) reviewed the data and an agreement was obtained. The risk of bias was rated for each study 


\section{Cureus}

by two authors independently, using the Cochrane criteria for randomized controlled trials (RCTs) [12]. Uncontrolled studies were always rated as moderate or high risk of bias (never at low risk of bias) and categorized as follows: moderate risk of bias, when the descriptions of patients, interventions and outcomes of interest were complete and clear throughout the observation period and high risk of bias, when the descriptions of patients, interventions and outcomes were incomplete or unclear, or when follow-up was incomplete.

The primary outcome analyzed in this study was spontaneous pleurodesis which was defined as a decrease in pleural fluid drainage to $<50 \mathrm{~mL}$ on three consecutive IPC drainage procedures and no evidence of significant effusion on chest ultrasonography, chest radiography, or chest computed tomography (CT) scan. The secondary outcomes included the time to achieve spontaneous pleurodesis, requirement of further pleural drainage after IPC placement and rate of complications (pleural fluid infection, pain at the insertion site, insertion-site cellulitis and pneumothorax).

All the studies included in this systematic review were observational, and hence, a meta-analysis could not be performed. Pooled results on the efficacy and safety outcomes were reported for each outcome as the percentage of patients with the primary or secondary outcome.

Findings were reported in tables and text, as data could not be pooled in forest plots.

\section{Results}

The search strategy described above retrieved a total of 370 published articles. Among these, only four case series were identified to qualify for inclusion, as described in Figure 1 . The characteristics of these primary studies are detailed in Table 1.

\begin{tabular}{|c|c|c|c|c|c|}
\hline \multirow[t]{5}{*}{ Study included } & Primary at & Time period of study & Year of publication & Country of population & Study design \\
\hline & Chalhoub et al. [13] & 2003-2009 & 2011 & United States of America & Case series \\
\hline & Bhatnagar et al. [14] & 2007-2013 & 2013 & United Kingdom & Case series \\
\hline & Sharaf-Eldin et al. [15] & Not reported & 2016 & Egypt & Case series \\
\hline & Chen et al. [16] & 2010-2015 & 2016 & United States of America & Case series \\
\hline
\end{tabular}

TABLE 1: Characteristics of primary studies

The characteristics of patients in the included studies are reported in Table 2. 


\section{Cureus}

\begin{tabular}{|c|c|c|c|c|c|}
\hline VARIABLES & Sharaf-Eldin et al. [15] & Chalhoub et al. [13] & Chen et al. [16] & Bhatnagar et al. [14] & Pooled Analysis \\
\hline Number of Patients & 60 & 8 & 24 & 19 & 111 \\
\hline Mean Age (years) & 42.3 & 56 & 59.8 & Not Reported & \\
\hline Male (n) & 36 & 6 & Not Reported & Not Reported & 42 out of $68(61.8 \%)$ \\
\hline Female (n) & 24 & 2 & Not Reported & Not Reported & 26 out of $68(38.2 \%)$ \\
\hline Right-sided Pleural Effusions (n) & Not Reported & 8 & 21 & Not Reported & 29 out of $32(90.6 \%)$ \\
\hline Left-sided Pleural Effusions (n) & Not Reported & 0 & 3 & Not Reported & 3 out of $32(9.4 \%)$ \\
\hline Child-Pugh A (n) & 0 & Not Reported & Not Reported & Not Reported & \\
\hline Child-Pugh B (n) & 32 & Not Reported & Not Reported & Not Reported & \\
\hline Child-Pugh C (n) & 28 & Not Reported & Not Reported & Not Reported & \\
\hline MELD Score & Not Reported & Not Reported & 19.4 & Not Reported & \\
\hline Alcoholic Cirrhosis (n) & Not Reported & Not Reported & 11 & Not Reported & \\
\hline NASH (n) & Not Reported & Not Reported & 6 & Not Reported & \\
\hline Chronic Hepatitis-induced Cirrhosis (n) & Not Reported & Not Reported & 6 & Not Reported & \\
\hline PBC Cirrhosis (n) & Not Reported & Not Reported & 1 & Not Reported & \\
\hline Cryptogenic Cirrhosis (n) & Report & Reporte & 1 & eport & \\
\hline
\end{tabular}

\section{TABLE 2: Characteristics of patients in included studies}

MELD: model for end-stage liver disease; NASH: nonalcoholic steatohepatitis; PBC: primary biliary cholangitis

In total, we investigated data from 111 patients. The mean age of patients was reported in three studies (Chalhoub et al. [13], Chen et al. [16] and Sharaf-Eldin et al. [15]) and ranged from 42 to 59 years. The majority of these patients were male (61.8\%). The location of pleural effusion was mentioned in two studies (Chalhoub et al. [13] and Chen et al. [16]) with right-sided predominance (90.6\%). Based on the available studies, it was difficult to estimate the duration of IPC in situ as it was not reported in most of the studies. The etiology of the underlying chronic liver disease was reported in only one study (Chen et al. [16]), with alcoholic cirrhosis (45\%) and nonalcoholic steatohepatitis (25\%) being the most common etiologies. Severity of the liver disease was also described only by Chen et al. [16], which included 32 patients with Child-Pugh class $\mathrm{B}$ cirrhosis and 28 patients with Child-Pugh class $\mathrm{C}$ cirrhosis. The mean number of thoracentesis before IPC placement was investigated in three studies (Chalhoub et al. [13], Chen et al. [16] and Bhatnagar et al. [14]) and ranged from 3.5 to 4.5 times.

The primary and secondary outcomes are reported in Table 3. 


\section{Cureus}

\begin{tabular}{|c|c|c|c|c|c|}
\hline VARIABLES & Sharaf-Eldin et al. [15] & Chalhoub et al. [13] & Chen et al. [16] & Bhatnagar et al. [14] & Pooled Analysis \\
\hline Mean number of thoracentesis before IPC (n) & Not reported & 3.5 & 1.9 & 4.5 & \\
\hline Spontaneous pleurodesis (n) & Not reported & 6 & 8 & 2 & 16 out of $51(31.4 \%)$ \\
\hline Mean time to spontaneous pleurodesis (days) & Not reported & 73.6 & 131.8 & 222 & \\
\hline Requirement of pleural drainage after IPC & Not reported & 0 & 0 & Not reported & 0 out of $32(0 \%)$ \\
\hline Pneumothorax (n) & 0 & 0 & 0 & Not reported & 0 out of $92(0 \%)$ \\
\hline Pain at insertion site (n) & 12 & Not reported & Not reported & Not reported & 12 out of $60(20 \%)$ \\
\hline Blockage of the catheter $(n)$ & 2 & 0 & Not reported & Not reported & 2 out of $68(2.9 \%)$ \\
\hline Pleural fluid infection (n) & 0 & 0 & 4 & 1 & 5 out of $111(4.5 \%)$ \\
\hline Reaccumulation after catheter removal (n) & 12 & 0 & Not reported & Not reported & 12 out of $68(17.6 \%)$ \\
\hline Catheter site cellulitis (n) & Not reported & 1 & 0 & Not reported & 1 out of $32(3.1 \%)$ \\
\hline Dissodgement $(\mathbf{n})$ & Not reported & 0 & Not reported & 1 & 1 out of $27(3.7 \%)$ \\
\hline
\end{tabular}

\section{TABLE 3: Primary and secondary outcomes}

IPC: indwelling pleural catheter

Of the 51 patients, 16 (31.4\%) achieved spontaneous pleurodesis. The mean duration to achieve spontaneous pleurodesis was described in three studies (Chalhoub et al. [13], Chen et al. [16] and Bhatnagar et al. [14]) and ranged from 73 to 22 days. Further requirement of pleural fluid drainage by other means after IPC placement was described by Chalhoub et al. [13] and Chen et al. [16]. In the 32 patients followed up, none of them required any drainage after IPC placement. As for secondary outcomes, the frequency of pneumothorax during or after the procedure was 0 out of 92 patients (0\%); pain at insertion site was reported in 12 out of 60 patients (20\%), catheter blockage in two out of 68 patients (2.9\%), pleural fluid infection in five out of 111 patients (4.5\%) and catheter-site cellulitis in one out of 32 patients (3.1\%). Reaccumulation of pleural fluid after catheter removal was mentioned in one study (Sharaf-Eldin et al. [15]), wherein 12 out of 60 patients (20\%) developed a recurrence of pleural effusion.

\section{Discussion}

The management of patients with cirrhosis and $\mathrm{HH}$ can be clinically challenging as limited objective data are available regarding the recommended treatment strategies for $\mathrm{HH}$. These patients are largely managed primarily with salt restriction and diuretics. In a case series by Badillo et al. [17], 83\% of patients with $\mathrm{HH}$ responded to sodium restriction and diuretics. Among patients with $\mathrm{HH}, 20 \%$ are refractory to diuretics, requiring other modalities for symptomatic control. While awaiting transplant, these options can include repeated therapeutic thoracentesis, pleurodesis, IPCs or TIPS. Patients with HH already have an overall elevated mortality rate compared to those with cirrhosis with the same MELD score [17]. Most patients with HH have Child-Pugh class B or class C cirrhosis and are listed for transplant; however, due to organ scarcity, alternative temporizing therapies are often needed [18-19].

An alternative to organ transplantation, TIPS also addresses the underlying pathology causing $\mathrm{HH}$ and can be an effective management strategy for recurrent HH [20]. Potential complications of TIPS include thrombosis or stenosis within the shunt. In addition, TIPS can cause hepatic encephalopathy in 15\% to 30\% of the patients [21]. Also, one-third of the patients continue to require repeated thoracentesis [21-22].

IPCs are widely used in patient populations with refractory pleural effusions secondary to malignancy, congestive heart failure and renal failure [14,23]. IPCs should be considered as a bridging treatment modality between liver transplantation and refractory HH because of its efficacy, safety and low cost.

We analyzed the rate of spontaneous pleurodesis as the primary outcome in our study. In smaller studies on $\mathrm{HH}$, the rate of pleurodesis ranged from $10 \%$ to $75 \%$ [13-14]. In our pooled analysis, pleurodesis was achieved in 16 out of 51 patients (31.4\%). This rate is comparable to the rate of pleurodesis achieved with IPCs in patients with congestive heart failure and malignant pleural effusions. In a systemic review by Patil et al. [24], 162 patients with refractory pleural effusion due to heart failure were managed with IPCs, of which 68 patients (42.1\%) achieved spontaneous pleurodesis. The rate of spontaneous pleurodesis in malignant pleural effusions was analyzed by Putnam at al. [25] and Davies et al. [26] and was found to be $46 \%$ and $51 \%$, respectively. The mean duration of achieving spontaneous pleurodesis in patients treated for HH with IPC 
ranged from 73 to 222 days, which is significantly higher than the time taken to achieve pleurodesis in malignant pleural effusions (36 days), as reported in a study by Van Meter et al. [23]. It is hypothesized that the longer time to achieve pleurodesis in $\mathrm{HH}$ could be secondary to rapid accumulation of fluid, which prevents the visceral and parietal pleural surfaces to approximate and adhere [27]. The mechanism of pleurodesis with IPC is not well understood, but it is thought to be related to low-grade inflammatory reaction that develops in response to the catheter acting as a foreign body in the pleural space [13].

As secondary outcomes, we analyzed the rate of pleural fluid infection, pneumothorax and catheter-site cellulitis after IPC insertion for HH. In a smaller study on HH by Fortin et al. [28], pleural fluid infection was overestimated to be $16.7 \%$, but as per our review, only five out of 111 patients (4.5\%) developed this complication. This rate of pleural fluid infection (4.5\%) was much lower than the frequency of pleural fluid infection reported by Davies et al. [26] in patients with malignant pleural effusions (13.4\%). However, the rate of pleural fluid infection after IPC placement for $\mathrm{HH}(4.5 \%)$ was found to be slightly higher compared to that in patients with congestive heart failure $(1.5 \%)$ and renal failure $(0 \%)[14,23]$. In our review, there were no reports of pneumothorax among 92 patients with HH. However, in patients with malignant pleural effusion requiring IPCs, the rate of pneumothorax requiring chest tube insertion was reported to be 5.9\% [29]. The rate of IPC blockage was also found to be lower in patients with $\mathrm{HH}(2.9 \%)$ as compared to those with malignant pleural effusion (3.7\%) [23]. Catheter blockage and insertion-site cellulitis were also found in only one of 32 cases (3.1\%) and 12 out of 60 patients (20\%), respectively, thus making IPCs a relatively safe therapeutic option for patients with $\mathrm{HH}$.

Among patients with refractory HH who are not yet able to receive a liver transplant, the use of IPCs may portend better survivals rates as compared to patients managed with TIPS. A review by Xiol et al. [30] presented 56 patients with $\mathrm{HH}$ undergoing liver transplantation with a one-year survival posttransplant of $82 \%$ and a mean survival of 97 months compared with 40 to $60 \%$ survival at 1 year and a mean survival of 7 to 13 months in patients with refractory HH treated with TIPS [31]. Moreover, in a case series by Campos et al. [20], TIPS was associated with $25 \%$ mortality at 30 days, and portosystemic encephalopathy was recorded in $66.6 \%$ of the cases. In addition to a higher reported mortality, TIPS has a high financial burden for the patient, as compared to an IPC. In a recent study by Penz et al. [32], the overall cost for managing patients with IPCs was estimated to be $\$ 4,993$ which is significantly lower than the expenditure for TIPS in which the mean hospital cost was $\$ 44,901 \pm \$ 54,565$ [33]. IPCs are a safe, economical and efficacious alternative to TIPS for patients who are awaiting liver transplantation and have recurrent $\mathrm{HH}$.

\section{Study limitations}

Our systematic review has several limitations. First, despite performing a comprehensive literature search in multiple well-established databases, independently conducted by two reviewers, and careful crossreferencing, we cannot exclude the possibility of having missed a relevant study. Second, there was large heterogeneity in the design of included studies and the reporting of results. Therefore, the number of studies that qualified for inclusion in each specific analysis was often small. Moreover, due to the absence of control groups in all the included studies, a meta-analysis could not be performed. In addition, although only this study provides the systematic review on this subject, a sample size of 111 is limited, which could underestimate the true rate of complications.

\section{Conclusions}

We conclude that IPCs are an acceptable therapeutic option for the management of refractory pleural effusion in patients with $\mathrm{HH}$, and possibly a superior option than TIPS. Although liver transplantation is the gold standard for the management of $\mathrm{HH}$ in these patients, availability is a limiting factor. Using IPCs for the management of refractory $\mathrm{HH}$ is a safe and efficacious therapy with a good rate of spontaneous pleurodesis.

\section{Additional Information}

\section{Disclosures}

Conflicts of interest: In compliance with the ICMJE uniform disclosure form, all authors declare the following: Payment/services info: All authors have declared that no financial support was received from any organization for the submitted work. Financial relationships: All authors have declared that they have no financial relationships at present or within the previous three years with any organizations that might have an interest in the submitted work. Other relationships: All authors have declared that there are no other relationships or activities that could appear to have influenced the submitted work.

\section{References}

1. Baikati K, Le DL, Jabbour II, Singhal S, Anand S: Hepatic hydrothorax. Am J Ther. 2014, 21:43-51. 10.1097/MJT.0b013e318228319e

2. Haas KP, Chen AC: Indwelling tunneled pleural catheters for the management of hepatic hydrothorax. Curr Opin Pulm Med. 2017, 23:351-356. 10.1097/MCP.0000000000000386

3. Singh A, Bajwa A, Shujaat A: Evidence-based review of the management of hepatic hydrothorax . Respiration. 2013, 86:155-73. 10.1159/000346996 
4. Orman ES, Lok AS: Outcomes of patients with chest tube insertion for hepatic hydrothorax. Hepatol Int. 2009, 3:582-6. 10.1007/s12072-009-9136-z

5. Liu LU, Haddadin HA, Bodian CA, Sigal SH, Korman JD, Bodenheimer HC Jr, Schiano TD: Outcome analysis of cirrhotic patients undergoing chest tube placement. Chest. 2004, 126:142-8. 10.1378/chest.126.1.142

6. Pfammatter R, Quattropani C, Reichen J, Göke B, Wagner AC: Treatment of hepatic hydrothorax and reduction of chest tube output with octreotide. Eur J Gastroenterol Hepatol. 2001, 13:977-80.

7. Borchardt J, Smirnov A, Metchnik L, Malnick S: Treating hepatic hydrothorax. BMJ. 2003, 326:751. 10.1136/bmj.326.7392.751

8. Chambers DM, Abaid B, Gauhar U: Indwelling pleural catheters for nonmalignant effusions: evidence-based answers to clinical concerns. Am J Med Sci. 2017, 354:230-235. 10.1016/j.amjms.2017.03.003

9. Moher D, Liberati A, Tetzlaff J, Altman DG: Preferred reporting items for systematic reviews and metaanalyses: the PRISMA statement. Int J Surg. 2010, 8:336-41. 10.1016/j.ijsu.2010.02.007

10. Loney PL, Chambers LW, Bennett KJ, Roberts JG, Stratford PW: Critical appraisal of the health research literature: prevalence or incidence of a health problem. Chronic Dis Can. 1998, 19:170-6.

11. The Newcastle-Ottawa Scale (NOS) for Assessing the Quality of Nonrandomised Studies in Meta-analyses by GA Wells, B Shea, D O'Connell, J Peterson, V Welch, M Losos, P Tugwell. (2016). Accessed: August 6, 2018: http://www.ohri.ca/programs/clinical_epidemiology/oxford.asp.

12. Higgins JPT: Cochrane Handbook for Systematic Reviews of Interventions Version 5.1.0 . Higgins JPT, Green Sally (ed): The Cochrane Collaboration, 2011.

13. Chalhoub M, Harris K, Castellano M, Maroun R, Bourjeily G: The use of the PleurX catheter in the management of non-malignant pleural effusions. Chron Respir Dis. 2011, 8:185-91. 10.1177/1479972311407216

14. Bhatnagar R, Reid ED, Corcoran JP, et al.: Indwelling pleural catheters for non-malignant effusions: a multicentre review of practice. Thorax. 2014, 69:959-61. 10.1136/thoraxjnl-2013-204563

15. Sharaf-Eldin M, Bediwy AS, Kobtan A, et al.: Pigtail catheter: a less invasive option for pleural drainage in Egyptian patients with recurrent hepatic hydrothorax. Gastroenterol Res Pract. 2016, 2016:10.1155/2016/4013052

16. Chen A, Massoni J, Jung D, Crippin J: Indwelling tunneled pleural catheters for the management of hepatic hydrothorax. A pilot study. Ann Am Thorac Soc. 2016, 13:862-6. 10.1513/AnnalsATS.201510-688BC

17. Badillo R, Rockey DC: Hepatic hydrothorax: clinical features, management, and outcomes in 77 patients and review of the literature. Medicine (Baltimore). 2014, 93:135-42. 10.1097/MD.0000000000000025

18. Siegerstetter V, Deibert P, Ochs A, Olschewski M, Blum HE, Rössle M: Treatment of refractory hepatic hydrothorax with transjugular intrahepatic portosystemic shunt: long-term results in 40 patients. Eur I Gastroenterol Hepatol. 2001, 13:529-34.

19. Lv Y, Han G, Fan D: Hepatic hydrothorax. Ann Hepatol. 2018, 17:33-46. 10.5604/01.3001.0010.7533

20. Campos S, Gomes D, Sofia C: Transjugular intrahepatic portosystemic shunt in refractory hydrothorax - a contribution to an unexplored indication. Eur J Gastroenterol Hepatol. 2016, 28:661-6. 10.1097/MEG.0000000000000623

21. Riggio O, Merlli M, Pedretti G, et al.: Hepatic encephalopathy after transjugular intrahepatic portosystemic shunt. Incidence and risk factors. Digest Dis Sci. 1996, 41:578-84. 10.1007/BF02282344

22. Zhuang ZW, Teng GJ, Jeffery RF, Gemery JM, Janne d'Othee B, Bettmann MA: Long-term results and quality of life in patients treated with transjugular intrahepatic portosystemic shunts. AJR Am J Roentgenol. 2002, 179:1597-603. 10.2214/ajr.179.6.1791597

23. Van Meter ME, McKee KY, Kohlwes RJ: Efficacy and safety of tunneled pleural catheters in adults with malignant pleural effusions: a systematic review. J Gen Intern Med. 2011, 26:70-6. 10.1007/s11606-0101472-0

24. Patil M, Dhillon SS, Attwood K, Saoud M, Alraiyes AH, Harris K: Management of benign pleural effusions using indwelling pleural catheters: a systematic review and meta-analysis. Chest. 2017, 151:626-35. 10.1016/j.chest.2016.10.052

25. Putnam JB Jr, Light RW, Rodriguez RM, et al.: A randomized comparison of indwelling pleural catheter and doxycycline pleurodesis in the management of malignant pleural effusions. Cancer. 1999, 86:1992-9. 10.1002/(SICI)1097-0142(19991115)86:10<1992::AID-CNCR16>3.0.CO;2-M

26. Davies HE, Mishra EK, Kahan BC, et al.: Effect of an indwelling pleural catheter vs chest tube and talc pleurodesis for relieving dyspnea in patients with malignant pleural effusion: the TIME2 randomized controlled trial. JAMA. 2012, 307:2383-9. 10.1001/jama.2012.5535

27. Ikard RW, Sawyers JL: Persistent hepatic hydrothorax after peritoneojugular shunt. Arch Surg. 1980, 115:1125-7. 10.1001/archsurg.1980.01380090091022

28. Fortin M, Tremblay A: Pleural controversies: indwelling pleural catheter vs. pleurodesis for malignant pleural effusions. J Thorac Dis. 2015, 7:1052-7. 10.3978/j.issn.2072-1439.2015.01.51

29. Michaud G, Barclay P, Tremblay A: Tunneled pleural catheters for palliation of malignant pleural effusions . J Bronchology. 2005, 12:245-8. 10.1097/01.lab.0000185782.99433.02

30. Xiol X, Tremosa G, Castellote J, Gornals J, Lama C, Lopez C, Figueras J: Liver transplantation in patients with hepatic hydrothorax. Transpl Int. 2005, 18:672-5. 10.1111/j.1432-2277.2005.00116.x

31. Strauss RM, Martin LG, Kaufman SL, Boyer TD: Transjugular intrahepatic portal systemic shunt for the management of symptomatic cirrhotic hydrothorax. Am J Gastroenterol. 1994, 89:1520-2.

32. Penz ED, Mishra EK, Davies HE, Manns BJ, Miller RF, Rahman NM: Comparing cost of indwelling pleural catheter vs talc pleurodesis for malignant pleural effusion. Chest. 2014, 146:991-1000. 10.1378/chest.132481

33. Kuei A, Lee EW, Saab S, et al.: Inpatient cost assessment of transjugular intrahepatic portosystemic shunt in the USA from 2001 to 2012. Dig Dis Sci. 2016, 61:2838-2846. 10.1007/s10620-016-4233-Z 